\title{
La obesidad materna durante el embarazo aumentaría el riesgo de muerte prematura en su descendencia
}

\author{
Maternal obesity during pregnancy may increase the risk of premature mortality in the offspring
}

Reynolds RM, et al. BMJ 2013;347:f4539

\section{Objetivo}

Determinar si la obesidad materna durante el embarazo aumenta el riesgo de muerte por todas las causas y los ingresos hospitalarios por eventos cardiovasculares en la descendencia en edad adulta.

\section{Diseño, lugar y pacientes}

Cohorte. Aberdeen, Escocia. Mujeres que dieron a luz un bebe único de término entre 1950 y 1976, y cuyo peso hubiera sido registrado en la primera visita del control prenatal, agrupadas en cuatro estratos según su Índice de Masa Corporal (IMC).

\section{Evaluación de factores pronósticos}

Se definieron como exposición la obesidad materna durante el embarazo, y como resultados principales la mortalidad por todas las causas y los ingresos hospitalarios por eventos cardiovasculares ${ }^{1}$ en la descendencia en edad adulta.

\section{Medición de resultados principales}

Se analizó el IMC materno como una variable categórica y luego como continua.

Se estimaron los errores estándar después de haber ajustado por la cantidad de hijos; y por posibles confundidores, incluyendo: edad materna en el parto, edad gestacional al momento de la primera medición del peso materno, clase social, paridad, sexo, edad gestacional al momento del parto, peso al nacer, y edad al momento de la muerte o evento cardiovascular.

Se analizaron a todas las mujeres y su descendencia; posteriormente solo a aquéllas pesadas en primera instancia antes de las 20 semanas de gestación; se investigó si la obesidad materna se asoció a la muerte prematura (menores de 55 años) de la descendencia; y posteriormente, en el subgrupo de los nacidos entre 1950 y 1955, se ajustó el análisis según el estatus socioeconómico de los hijos.

\section{Resultados Principales}

Se seleccionaron a 28.540 mujeres. Las mujeres obesas (alrededor del $4 \%$ ) eran mayores, de mayor paridad, de clase social más baja y con el primer registro de peso más tardío. Los hijos nacidos de madres obesas tuvieron mayor peso de nacimiento, pero menor edad gestacional. De los 37.709 hijos, se registraron 6.551 muertes por todas las causas.

Se observó un aumento del riesgo de muerte prematura por todas las causas y por eventos cardiovasculares, así como de las admisiones hospitalarias por eventos cardiovasculares. Ver Tabla 1.

Tabla 1. Muertes por eventos cardiovasculares según IMC materno, tomando como control al grupo de madres con normopeso.

\begin{tabular}{|c|c|c|}
\hline & Muertes (\%) & HR (IC 95\%) ajustado \\
\hline \multicolumn{3}{|c|}{ Descendencia de todas las madres } \\
\hline Madre sobrepeso & $1.337(20,4)$ & $1,17(1,08$ a 1,28$)$ \\
\hline Madre obesa & $294(4,5)$ & $1,42(1,19$ a 1,69$)$ \\
\hline \multicolumn{3}{|c|}{ Hijos menores de 55 años } \\
\hline Madre sobrepeso & $805(18,4)$ & $1,19(1,09$ a 1,30$)$ \\
\hline Madre obesa & $152(3,5)$ & $1,40(1,17$ a 1,68$)$ \\
\hline
\end{tabular}

HR: hazard ratio.

\section{Conclusiones}

La obesidad materna durante el embarazo se asocia a un aumento del riesgo de muerte prematura por todas las causas de su descendencia, independientemente del estatus socioeconómico. Dada la alta prevalencia de obesidad, los resultados subrayarían la necesidad imperiosa de desarrollar estrategias para optimizar el peso de las mujeres en edad reproductiva, así como el asesoramiento acerca del riesgo cardiovascular de sus hijos.

Fuente de financiamiento: Chief Scientist Office, Scotland; Chest, Heart and Stroke, Scotland. Tommy's and the British Heart Foundation.

\section{Comentario}

Si bien el estudio realiza un análisis ajustado a confundidores, no se hace referencia a posibles variaciones en cuanto al estilo de vida de la descendencia, incluyendo al tabaquismo, alcoholismo, sedentarismo, drogadicción, hipertensión y dislipemia. Sin embargo, dada la alta prevalencia de obesidad en mujeres en edad reproductiva, como también de la muerte temprana por eventos cardiovasculares en Argentina $^{2}$ y el resto del mundo ${ }^{3,4}$; este trabajo resultaría muy interesante para tener en consideración la ampliación del espectro de población blanco de la aplicación de políticas sanitarias que apunten a los cambios en el estilo de vida y la prevención de la obesidad, más allá de las repercusiones posibles en el riesgo obstétrico en sí mismo ${ }^{5}$; como así también, para continuar enfatizando la importancia de la promoción de los cambios en el estilo de vida como medio fundamental de la prevención primaria en nuestra práctica cotidiana.

Cecilia Laura Drimer [ Servicio de Medicina Familiar y Comunitaria del Hospital Italiano de Buenos Aires. cecilia.drimer@hospitalitaliano.org.ar ]

Drimer C. La obesidad materna durante el embarazo aumentaría el riesgo de muerte prematura en su descendencia. Evid Act Pract Ambul. Ene-Mar 2014;17(1):17. Comentado de: Maternal obesity during pregnancy and premature mortality from cardiovascular event in adult offspring: follow-up of 1323275 person years. Reynolds RM, et al. BMJ 2013;347:f4539 doi: 10.1136/bmj.f4539. PMID: 23943697.

\section{Referencias}

1.Organización Mundial de la Salud. Clasificación Internacional de Enfermedades (en inglés ICD) [consultado 16 Noviembre de 2013]. Disponible en:http://www.who.int/classifications/icd/en/

2. Organización Mundial de la Salud - ENT Perfiles de países. [actualizado 2011; consultado 16 Noviembre de 2013]. Disponible en:http://www.who.int/nmh/countries/arg_es.pdf 3. Flegal KM, Carroll MD, Kit BK, Ogden CL. Prevalence of obesity and trends in the distribution of body mass index among US adults, 1999-2010. JAMA 2012;307:491-7.

4. Heslehurst N, Rankin J, Wilkinson JR, Summerbell CD. A nationally representative study of maternal obesity in England, UK: trends in incidence and demographic inequalities in 619323 births, 1989-2007. Int J Obes (Lond) 2010;34:420-8.

5. Chu S.Y, et al. Association between Obesity during Pregnancy and Increased Use of Health Care. N Engl J Med 2008;358:1444-53. 